\title{
A New Digital Signal Processing Method for Spectrum Interference Monitoring
}

\author{
L. Angrisani ${ }^{1}$, D. Capriglione ${ }^{2}$, L. Ferrigno ${ }^{2}$, G. Miele $^{2}$ \\ ${ }^{1}$ Department of Computer Science and Control Systems, Faculty of Engineering, University of Naples "Federico II", \\ via Claudio, 21, 80125, Napoli, Italy, angrisan@unina.it \\ ${ }^{2}$ DAEIMI, Faculty of Engineering, University of Cassino, via G. Di Biasio, 43, 03043, Cassino (FR), Italy, \\ \{capriglione,ferrigno,g.miele@unicas.it\}
}

\begin{abstract}
Frequency spectrum is a limited shared resource, nowadays interested by an ever growing number of different applications. Generally, the companies providing such services pay to the governments the right of using a limited portion of the spectrum, consequently they would be assured that the licensed radio spectrum resource is not interested by significant external interferences. At the same time, they have to guarantee that their devices make an efficient use of the spectrum and meet the electromagnetic compatibility regulations. Therefore the competent authorities are called to control the access to the spectrum adopting suitable management and monitoring policies, as well as the manufacturers have to periodically verify the correct working of their apparatuses. Several measurement solutions are present on the market. They generally refer to real-time spectrum analyzers and measurement receivers. Both of them are characterized by good metrological accuracies but show costs, dimensions and weights that make no possible a use "on the field". The paper presents a first step in realizing a digital signal processing based measurement instrument able to suitably accomplish for the above mentioned needs. In particular the attention has been given to the DSP based measurement section of the instrument. To these aims an innovative measurement method for spectrum monitoring and management is proposed in this paper. It performs an efficient sequential analysis based on a sample by sample digital processing. Three main issues are in particular pursued: (i) measurement performance comparable to that exhibited by other methods proposed in literature; (ii) fast measurement time, (iii) easy implementation on cost-effective measurement hardware.
\end{abstract}

Keywords: Spectrum monitoring, spectrum management, spectrogram, digital signal processing, RF measurement

\section{INTRODUCTION}

$\boldsymbol{F}^{\mathrm{R}}$ REQUENCY SPECTRUM is a limited resource and the demand for its use is nowadays increasing. Many governments in the world, recognizing its market value, began auctioning the right to use their airwaves. Companies that purchase spectrum license, offer a wide range of for-fee services such as voice calls, text messaging, wireless Internet, High-Definition Television (HDTV) and so on. For service providers and equipment manufacturers, sums of money are at stake in the production and delivery of wireless products and services. Extensive regulatory requirements have been designed to avoid unwanted interference between users that share the radio spectrum resource. Special groups routinely monitor emissions to ensure that transmission equipments comply with regulations. Compliance monitoring of signal spectrum is continuously increasing.

The growth of commercial signal monitoring applications has increased significantly with the diffusion of wireless devices [1]. Inadvertent interfering emissions can be very costly to cellular network operators. Likewise, commercial broadcasters can lose substantial market audiences due to a poorly controlled adjacent channel station. Furthermore the $\mathrm{RF}$ spectrum is continually getting more crowded. More consumer devices are starting to communicate with one another, and computer networking is expanding at a phenomenal rate [1]. All of these competing spectrum users must coexist without serious interference. In such RF environment, all equipment must meet spurious emission requirements to allow a clean path to neighboring signals.
Determining fault in interference cases begins with monitoring of transmitted spectral emissions [2]. Spectrum regulators need to monitor different types of signals to determine if enforcement actions are warranted [3].

As a result, the need of spectrum measurement and monitoring is becoming more and more mandatory. To these aims, several measurement solutions are proposed. They are based on two main classes of instruments: (a) real-time spectrum analyzers (RSAs) and (b) measurement receivers.

The former can be considered as an advanced version of the standard Vector Signal Analyzers (VSAs), specifically addressed to the analysis of complex digitally modulated signals. They are mainly used for general-purpose wireless spectrum monitoring applications. In addition, thanks to their special hardware architecture, they can perform a high number of Discrete Fourier Transforms (DFTs) in a very short time as to guarantee both a seamless acquisition and the estimation of the spectrogram [4], which correlates time and frequency information. However the cost, dimensions and weight of such instruments may limit their use, especially for on-field applications. Differently from RSAs, measurement receivers are specifically addressed to a target application, for example a particular communication standard. This turns out into proper features, such as built-in "personalities", mandated to warrant standards-compliant measurements of wireless formats [5]. Moreover, newer solutions complemented with additional DSP-based hardware are now appearing on the market, capable of providing, as RSAs do, a spectrogram analysis. They exhibit attractive weight, size and dimensions, even though showing 
worse accuracy than that peculiar to RSAs [6], [7].

Stemming from their past experience in the field of power and spectrum measurements of digital communication signals [8], [9], the authors propose a new method for spectrum monitoring and management entitled to be a valid, cost-effective alternative to the abovementioned solutions. Three main issues are in particular pursued: (i) easy implementation on cost-effective DSP (Digital Signal Processing) or FPGA (Field Programmable Gate Array) hardware, (ii) measurement performance, in terms of accuracy, resolution and sensitivity, comparable to that exhibited by RSAs; and (iii) measurement time lower than those characterizing RSAs.

The proposed method relies on a sequential approach based on a sample-by-sample processing. It could overcome typical limits that ordinary solutions, performing a batch processing on fixed-length overlapped segments of data, like those based on FFTs or Short-Time Fourier Transforms (STFTs), suffer from.

In the following, after a brief description of the proposed measurement method, a tuning stage is performed and, finally, some experiments on frequency hopping signals are presented.

\section{THEORETICAL BACKGROUND}

Traditional power spectrum density (PSD) estimation methods can be classified in two categories: nonparametric and parametric. Parametric methods can exhibit a reduced convergence time and are entitled to provide more significant results than those achievable from nonparametric approaches when the acquired record covers a relatively short time interval. Furthermore they can be implemented in an optimized manner (sequential estimation), thus allowing measurement results to be updated whenever a new sample is available and removing the need to locally store a large number of acquired samples [8]. Thanks to these characteristics they can be considered as good solution to be implemented in cost-effective hardware platforms [10], [11].

Among the several parametric PSD estimation methods the widespread used are the autoregressive (AR) estimation methods [8]-[11]. They suppose that the analyzing signal is the output of a linear system as specified in the following:

$$
x(n)=-\sum_{m=1}^{p} a_{p, m} x(n-m)+\varepsilon(n)
$$

where $x(n)$ is the analyzed signal sample at the time interval $n, a_{p, 1}, a_{p, 2}, \ldots, a_{p, p}$ are the model coefficients, $\{\varepsilon(n)\}$ is a white noise process with variance $\sigma_{p}^{2}$, and $p$ is the model order. The PSD of a signal modeled in this way is totally described by the model parameters and the variance of the white noise process

$$
S(f)=\frac{\sigma_{p}^{2} T_{S}}{\left|1+\sum_{m=1}^{p} a_{p, m} e^{-j 2 \pi m f T_{s}}\right|^{2}} \quad|f| \leq f_{N}
$$

where $T_{S}=1 / f_{S}$ is the sampling interval and $f_{N}=1 /\left(2 T_{S}\right)$ is the Nyquist rate.

Consequently, known $p$, it is necessary to properly estimate the $p+1$ parameters $a_{p, 1}, a_{p, 2}, \ldots, a_{p, p}$ and $\sigma_{p}^{2}$.

The most popular approach for AR parameter estimation with N-1 data samples was introduced by Burg in 1967 [12]. It estimates the model parameters for order $i$ starting from those previously estimated for order $i-1$ by calculating the reflection coefficients $a_{i, i}$ [13] which minimize a sum of forward and backward linear prediction error energies:

$$
S S_{i}(N-1)=\sum_{n=i}^{N-1}\left[\left|e_{i}(n)\right|^{2}+\left|b_{i}(n)\right|^{2}\right] \text { for } 1 \leq i \leq p
$$

where $N-1$ is the index corresponding to the actual discrete sample time, $e_{i}(n)$ is the forward linear prediction error at order $i, b_{i}(n)$ is the backward linear prediction error at order $i$, whose expressions are:

$$
e_{i}(n)=\sum_{k=0}^{i} a_{i, k} x(n-k) \text { for } 1 \leq i \leq p \text { and } i \leq n \leq N-1
$$

and

$b_{i}(n)=\sum_{k=0}^{i} a_{i, k} x(n-i+k)$ for $1 \leq i \leq p$ and $i \leq n \leq N-1$.

Note that $a_{p, 0}$ is defined as unity.

The AR parameters are computed using the so called Levinson-Durbin recursions [13]:

$$
\begin{gathered}
a_{i, m}=a_{i-1, m}+a_{i, i} a_{i-1, i-m}, \quad 1 \leq m \leq i-1 \quad 1 \leq i \leq p \\
\sigma_{i}^{2}=\sigma_{i-1}^{2}\left(1-\left|a_{i, i}\right|^{2}\right) \quad 1 \leq i \leq p .
\end{gathered}
$$

Consequently it is only important to estimate $a_{i, i}$, for all model order from $i=1 \ldots p$.

Substituting (4) and (5) into (3) and using (6), it is possible to demonstrate that $S S_{i}$ depends only by $a_{i, i}$, and it can be minimized by imposing:

$$
a_{i, i}=K_{i}(N-1)=-\frac{2 \sum_{n=i}^{N-1} e_{i-1}(n) b_{i-1}(n-1)}{\sum_{n=i}^{N-1}\left[\left|e_{i-1}(n)\right|^{2}+\left|b_{i-1}(n-1)\right|^{2}\right]}
$$

Equation (8) in combination with (6) and (7), for $i=1, \ldots, p$ forms a recursive algorithm for the PSD estimation. Even thought this method is characterized by good metrological performance, it is not suitable to be implemented on costeffective platforms, because, operating on batch data, it asks a huge memory requirement and a computational burden not compatible with cost-effective hardware platforms [11]. Fortunately a time-update recursive formulation for (8) is given by [14] and it is reported in the top of the following page. 


$$
K_{i}(N)=K_{i}(N-1)-\frac{\left[K_{i}(N-1)\left(\left|e_{i-1}(N)\right|^{2}+\left|b_{i-1}(N-1)\right|^{2}\right)+2 e_{i-1}(N) b_{i-1}(N-1)\right]}{\sum_{n=i}^{N}\left[\left|e_{i-1}(n)\right|^{2}+\left|b_{i-1}(n-1)\right|^{2}\right]} \text { for } 1 \leq i \leq p
$$

This new equation in combination with (6) and (7), for $i=1, \ldots, p$ and with initial conditions $e_{0}(N)=b_{0}(N)=x(N)$, forms a sequential time-update algorithm for the reflection coefficients.

This is the sequential Burg algorithm and it can update the PSD estimate whenever a new sample is available and it is characterized by a good trade-off between metrological performance and hardware requirements [11]. Unfortunately it is affected by an infinite memory, and doesn't track the PSD time evolution. In this way the output of its analysis is a snap-shot of what it is happened during the total observation period. This effect is clearly shown in the section 4 in which some numerical results are given. A possible solution to reduce this effect might be to reset the algorithm output in prearranged time intervals. This solution shows some limits related to the length of the observation period, which it should be short enough to warrant an adequate spectrum tracking, and at the same time long enough to warrant the convergence of the sequential estimation. Inaccuracies and loss of repeatability may be otherwise observed. Therefore alternative solutions have to be considered.

\section{The Proposed Method}

As previously described, an effective spectrum monitoring requires suitable PSD time tracking. This means that it is necessary to implement a measurement method able to produce a new PSD estimation on a very short observation time and of reducing the influence of previous acquired samples respect to the recent ones. To this aim, two hypotheses have been done: the former adopts a fixed-length sliding window, the latter an exponentially growing window [15].

The fixed-length sliding window permits considering only a finite number of past data values. Even though it seems to be effective, its implementation requires to store in the memory as many past input values as are included in the window length.

The exponentially growing window weighs the input samples giving more importance to the recent samples and attenuating the effects of the past ones (see Fig.1b). This window does not need to store samples in memory, thus resulting more proper for development on cost-effective hardware. Then, in the following this second approach is adopted, by modifying the traditional Burg cost function (3) in this way:

$$
S S_{i}(N-1)=\sum_{n=i}^{N-1} \lambda^{N-1-n}\left[\left|e_{i}(n)\right|^{2}+\left|b_{i}(n)\right|^{2}\right] \text { for } 1 \leq i \leq p
$$

where $\lambda$, termed forgetting factor, is in the following range $0<\lambda<1$. Note that $\lambda=1$ results in the traditional Burg case. Typical values of $\lambda$ are included in the range from 0.9 to 1 and it should be carefully selected to achieve the required performance [15].

It is possible to demonstrate [14] that, analogously to the sequential Burg algorithm, (10) can be minimized by imposing:

$$
K_{i}(N-1)=-\frac{2 \sum_{n=i}^{N-1} \lambda^{N-1-n} e_{i-1}(n) b_{i-1}(n-1)}{D E N_{i}(N-1)} \text { for } 1 \leq i \leq p
$$

where

$$
\operatorname{DEN}_{i}(N-1)=\sum_{n=i}^{N-1} \lambda^{N-1-n}\left[\left|e_{i-1}(n)\right|^{2}+\left|b_{i-1}(n-1)\right|^{2}\right] \text { for } 1 \leq i \leq p
$$

A time-update recursive formulation for (11) is reported in the bottom of this page, in which $N$ denotes the actual time index.
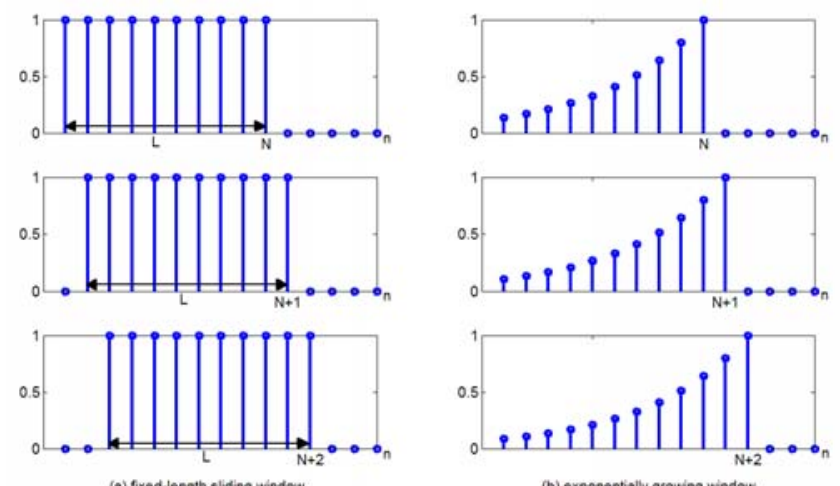

Fig.1. Example of a L-length sliding window (a) and an exponentially growing window (b).

Equation (13) in combination with the Levinson-Durbin recursions, for $i=1, \ldots, p$ and with initial conditions $e_{0}(N)=b_{0}(N)=x(N)$, forms the modified sequential Burg timeupdate algorithm for the reflection coefficients.

The proposed method operates as follows: after a preliminary initialization stage, every time a new sample is acquired a new loop starts. For each iteration of the loop it updates the model parameters by using (6) and (7), the reflection coefficients $K_{i}$ and the prediction errors $e_{i}$ and $b_{i}$ using (13) and (4), (5) respectively. The loop is composed by a number of iterations equal to the model order $p$. When the loop is ended a new PSD of the analyzing signal is suitably estimated by using the equation (2).

$$
K_{i}(N)=K_{i}(N-1)-\frac{\left[K_{i}(N-1)\left(\left|e_{i-1}(N)\right|^{2}+\left|b_{i-1}(N-1)\right|^{2}\right)+2 e_{i-1}(N) b_{i-1}(N-1)\right]}{\lambda D E N_{i}(N-1)+\left|e_{i-1}(N)\right|^{2}+\left|b_{i-1}(N-1)\right|^{2}} \quad \text { for } 1 \leq i \leq p
$$


For each acquired sample the proposed method requires $9 p$ real multiplications and $7 p$ real additions if only the reflection coefficients are updated with a consequent computational burden equal to $16 p$ floating point operations (flops). If the estimates of the AR parameters have to be updated the application of the Levinson-Durbin recursions requires $p^{2}$ real multiplications and $p^{2}$ real additions, increasing the computational burden to $2 p^{2}+16 p$ flops [10].

\section{TUNING OF THE METHOD}

As described in the previous section, the value of the forgetting factor $\lambda$ has to be suitably selected. To choose the optimal value of $\lambda$ a preliminary experimental test campaign performed in Matlab $7^{\mathrm{TM}}$ simulation environment has been performed. In particular, the proposed measurement method has been adopted to track a disturbing signal that changes its frequency characteristics during the observation period.

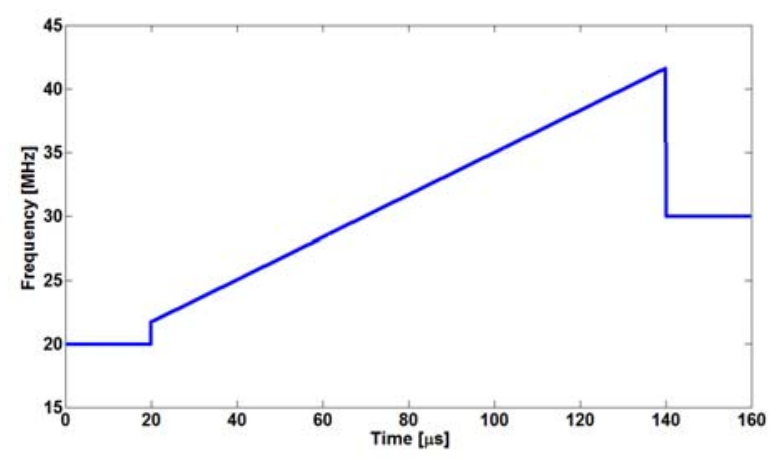

Fig.2. Frequency-time behaviour of the test signal.

To this aim, a special test signal characterized by the frequency behavior reported in Fig. 2 has been designed, in order to emulate typical test cases that are possible to find in real applications. It is composed by a single frequency tone that for the first $20 \mu \mathrm{s}$ is equal to $20 \mathrm{MHz}$, successively it hops at $21.7 \mathrm{MHz}$ and starts a linear frequency sweep reaching $41.6 \mathrm{MHz}$ in $120 \mu \mathrm{s}$. At the end it makes a large hop to $30 \mathrm{MHz}$, stabilizing on this value for the last $20 \mu \mathrm{s}$.

As for the measurement method, a sampling rate equal to $100 \mathrm{MS} / \mathrm{s}$ and a vertical resolution equal to 7 bit effective [10] have been considered. The spectrogram has been evaluated considering 4096 bins for each spectrum, obtaining a frequency resolution equal to $24 \mathrm{kHz}$.

Previous studies on Orthogonal frequency-division multiplexing (OFDM) signals (that can be view as sum of equally-spaced frequency tones) inside the frequency range of the considered test signal and sampled at $100 \mathrm{MS} / \mathrm{s}$ have suggested a model order $p$ equal to 40 to be selected to provides a good PSD estimation [10], [11]. The following figures of merit have been analyzed:

(i) the settling time $\left(t_{\text {set }}\right)$. It represents a convergence time and is evaluated as the mean time required to coerce the percentage frequency error (difference between the estimated and the imposed frequency divided by the imposed frequency, expressed in percentage) inside an area bounded by a threshold equal to $\pm 1.5 \%$; (ii) the mean convergence frequency error $\left(\mu_{e f}\right)$ defined as the mean frequency error evaluated after the settling time;

(iii) the standard deviation of the convergence frequency error $\left(\sigma_{e f}\right)$ defined as the standard deviation of the frequency error evaluated after the settling time.

All the figures of merit are evaluated for different values of $\lambda$ ranging in its typical interval, from 0.9 to 1 , (see [15]).

As regard the figure of merit (i), it has been evaluated at three different interesting points:

1) at the start time $\left(t_{s e t, i n}\right)$, in order to estimate the initial transient of the algorithm;

2) when the first frequency hop happens and the signal starts its frequency sweep $\left(t_{\text {set, } A}\right)$. This information is useful because it allows estimating how the proposed method reacts to a sharp frequency variation followed by a smooth one;

3) when the second hop happens and the signal frequency is settled at $30 \mathrm{MHz}\left(t_{\text {set, }, B}\right)$. With this analysis it is possible to estimate the method behavior after a very sharp frequency variation.

The measurement results related to the settling time analysis are reported in Table I. This figure of merit was not evaluated when $\lambda=1$ was involved because it is not able to track the signal frequency.

Table I. Settling Time Versus Forgetting Factor.

\begin{tabular}{|c|c|c|c|}
\hline \multirow{2}{*}{$\begin{array}{c}\text { Forgetting } \\
\text { factors } \lambda\end{array}$} & \multicolumn{3}{|c|}{ Settling time } \\
\cline { 2 - 4 } & $\boldsymbol{t}_{\text {set,in }}[\mathbf{n s}]$ & $\boldsymbol{t}_{\text {set, } \boldsymbol{A}}[\boldsymbol{\mu} \mathbf{s}]$ & $\boldsymbol{t}_{\text {set, } \boldsymbol{B}}[\boldsymbol{\mu s}]$ \\
\hline$\lambda=0.999$ & 280 & 19.38 & 1.05 \\
\hline$\lambda=0.998$ & 280 & 9.23 & 0.80 \\
\hline$\lambda=0.996$ & 280 & 3.47 & 1.14 \\
\hline$\lambda=0.994$ & 280 & 1.59 & 0.83 \\
\hline$\lambda=0.992$ & 280 & 1.00 & 0.65 \\
\hline$\lambda=0.990$ & 280 & 0.83 & 0.55 \\
\hline$\lambda=0.980$ & 330 & 51.29 & 0.28 \\
\hline$\lambda=0.960$ & 380 & 98.89 & 14.65 \\
\hline$\lambda=0.940$ & 11890 & 99.87 & 19.99 \\
\hline$\lambda=0.920$ & 14980 & 100 & 20.00 \\
\hline$\lambda=0.900$ & 14990 & 100 & 19.99 \\
\hline
\end{tabular}

From the analysis of the obtained measurement results it is possible to highlight that:

a) $t_{\text {set,in }}$ is almost constant and it does not seem to be influenced by the forgetting factor, $\lambda$, in the interval 0.990 0.999 . Outside this interval $t_{s e t, i n}$ starts noticeably to increase, reaching values near to $15 \mu$ s at $\lambda=0.900$;

b) $t_{s e t, A}$ and $t_{s e t, B}$ are influenced by the forgetting factor value, in particular the proposed method seems to reduce its convergence time when $\lambda$ value decreases from 0.999 to 0.99. Outside this interval it possible to note a reversal;

c) $t_{s e t, B}$ values are ever lower than $t_{s e t, A}$ ones, demonstrating that the even though this situation is characterized by a high frequency hop, the final frequency remains constant, making easier its tracking by the proposed method;

d) values of $\lambda$ lower than 0.940 show a $t_{s e t, B}$ equal to $20 \mu \mathrm{s}$, i.e. equal to duration of the $30 \mathrm{MHz}$-tone, demonstrating an insufficient ability to track the signal. 
Thanks to these consideration only the values of $\lambda$ included in the interval $0.990-0.999$ was considered in the subsequent stages of the work.

As regard the figure of merits (ii) and (iii), they have been evaluated in the following time intervals:

1) $t_{\text {set,in }}<\mathrm{t}<20 \mu \mathrm{s}$, in order to estimate the frequency tracking performance of the proposed method after the starting transient and in presence of a single tone;

2) $t_{s e t, A}<\mathrm{t}<140 \mu \mathrm{s}$. This analysis allows evaluating the measurement performance in presence of a swept-frequency tone, after a short transient caused by a little frequency hop;

3) $t>t_{s e t, B}$, allows analyzing the method performance in presence of a single tone after a deep frequency hop.

Table II. Measurement Performance at Steady-State. Mean Convergence Frequency Error and Standard Deviation of the Convergence Frequency Error Versus Forgetting Factor.

\begin{tabular}{|c|c|c|c|c|c|c|}
\hline \multirow{3}{*}{$\begin{array}{l}\text { Forgetting } \\
\text { factors } \lambda\end{array}$} & \multicolumn{2}{|c|}{$t_{\text {set, } i n}<t<20 \mu s$} & \multicolumn{2}{|c|}{$t_{s e t, A}<t<140 \mu s$} & \multicolumn{2}{|c|}{$t>t_{s e t, B}$} \\
\hline & $\mu_{e f}$ & $\sigma_{e f}$ & $\mu_{e f}$ & $\sigma_{e f}$ & $\mu_{e f}$ & $\sigma_{e f}$ \\
\hline & \multicolumn{2}{|c|}{ [kHz] } & \multicolumn{2}{|c|}{ [kHz] } & \multicolumn{2}{|c|}{ [kHz] } \\
\hline$\lambda=0.999$ & 26 & 46 & -95 & 40 & 22 & 12 \\
\hline$\lambda=0.998$ & 27 & 47 & -74 & 81 & 18 & 13 \\
\hline$\lambda=0.996$ & 21 & 51 & -73 & 96 & 23 & 23 \\
\hline$\lambda=0.994$ & 20 & 57 & -77 & 93 & 27 & 34 \\
\hline$\lambda=0.992$ & 25 & 68 & -61 & 92 & 21 & 44 \\
\hline$\lambda=0.990$ & 66 & 77 & -72 & 84 & 36 & 58 \\
\hline
\end{tabular}

From the analysis of the measurement results, reported in Table II, the following considerations can be drawn:

a) the mean frequency error obtained when the analyzing signal is a single tone at a fixed frequency is ever lower than $66 \mathrm{kHz}$, that corresponds to $0.33 \%$;

b) the mean frequency error experienced during the linear frequency sweep is ever higher than one evaluated when a simple tone is applied;

c) the experimental standard deviation $\left(\sigma_{e f}\right)$ seems to be influenced in inverse proportion by $\lambda$, it is important to consider that the frequency resolution of the analysis is $24 \mathrm{kHz}$;

d) a value of $\lambda$ inside the range 0.992-0.998 seems to grant a good trade-off between measurement accuracy and convergence time.

In Fig.3, as an example, the obtained spectrograms for three values $\lambda$ are reported. In particular the values considered in Figure 3 are $\lambda=1.000$ (absence of forgetting) $\lambda=0.996$ and $\lambda=0.900$. The information related to the amplitude of the PSD is coded by the color and the measurement unit is $\mathrm{dBm}$.

From the analysis of these results the following consideration can be drawn:

a) all the measures are characterized by a good signal to noise ratio (SNR);

b) measurement results, obtained when $\lambda=1$ is involved, are affected by the memory effect that does not allow a good frequency tracking;

c) a value of $\lambda$ equal 0.900 seems to be not adequate for this analysis, showing an instability in frequency tracking performance;

d) an intermediate value of $\lambda$ equal to 0.996 seems to present negligible memory effects and a good frequency tracking performance.

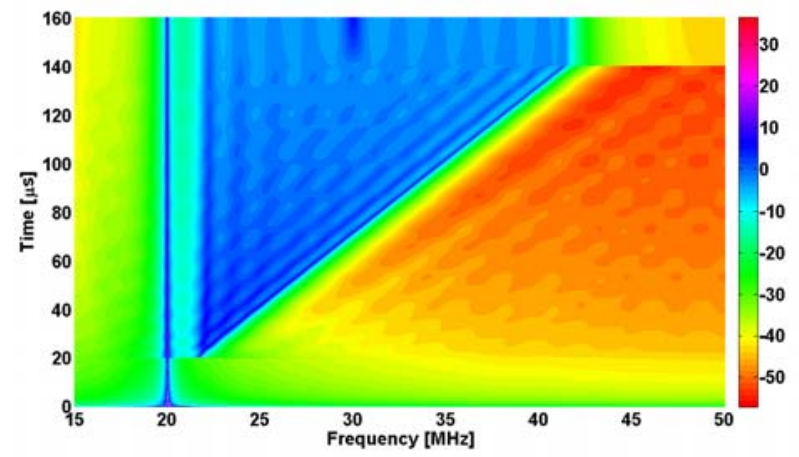

(a) $\lambda=1.000$

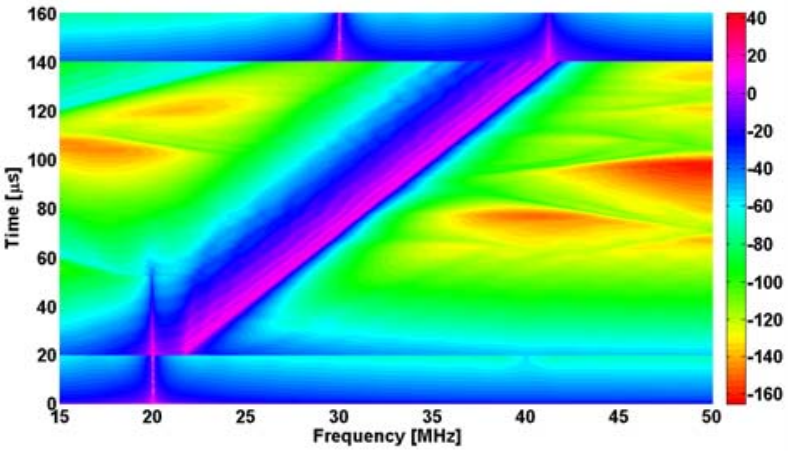

(b) $\lambda=0.996$

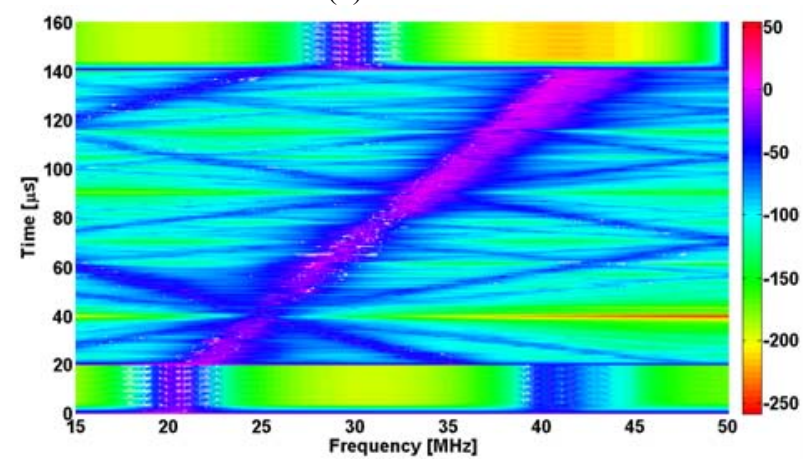

(c) $\lambda=0.900$

Fig.3. The obtained spectrograms, evaluated considering three values of $\lambda$.

\section{Performance ASSESSMENT}

To verify the measurement method performance further tests have been carried out on a frequency hopping signal. This class of signals has been selected as test set since can be considered as the worst case operating of the measurement method due to frequent and rapid variations that characterize these signals. In the author opinion they operate as step signals do in the characterization of a dynamic system where they highlight time responses and metrological characteristics.

More in detail, the test signal has been designed to evaluate the method performance when the analyzing signal perform a very large hop followed by a very short one. In fact it is composed by a single frequency tone that for the 
first $40 \mu$ s is equal to $10 \mathrm{MHz}$, successively it makes a large hop to $20 \mathrm{MHz}$ and remains stable at this frequency for 40 $\mu \mathrm{s}$. Afterwards it hops at $15 \mathrm{MHz}$ and after $40 \mu \mathrm{s}$ it makes a new small hop at $16 \mathrm{MHz}$ stabilizing on this value for the last $40 \mu \mathrm{s}$.

As for the measurement method, the same test conditions and figures of merit illustrated in the previous section have been taken into account. As in the previous section the figures of merit have been evaluated at the start time $\left(t_{\text {set,in }}\right)$ and after each hop $\left(t_{s e t, A}, t_{s e t, B}\right.$ and $\left.t_{s e t, C}\right)$, considering different values of $\lambda$ ranging from 0.99 to 0.999 .

Table III. Settling Time Versus Forgetting Factor.

\begin{tabular}{|c|c|c|c|c|}
\hline \multirow{2}{*}{$\begin{array}{c}\text { Forgetting } \\
\text { factors } \lambda\end{array}$} & \multicolumn{4}{|c|}{ Settling time } \\
\cline { 2 - 5 } & $\boldsymbol{t}_{\boldsymbol{s e t}, \boldsymbol{i n}}[\mathbf{n s}]$ & $\boldsymbol{t}_{\boldsymbol{s e t}, \boldsymbol{A}}[\boldsymbol{\mu} \mathbf{s}]$ & $\boldsymbol{t}_{\boldsymbol{s e t}, \boldsymbol{B}}[\boldsymbol{\mu} \boldsymbol{s}]$ & $\boldsymbol{t}_{\boldsymbol{s e t}, \boldsymbol{C}}[\boldsymbol{\mu} \mathbf{s}]$ \\
\hline$\lambda=0.999$ & 710 & $\mathrm{~N} / \mathrm{A}$ & $\mathrm{N} / \mathrm{A}$ & $\mathrm{N} / \mathrm{A}$ \\
\hline$\lambda=0.998$ & 710 & 8.87 & 21.56 & 9.26 \\
\hline$\lambda=0.996$ & 760 & 1.51 & 3.87 & 5.51 \\
\hline$\lambda=0.994$ & 860 & 0.93 & 1.13 & 1.56 \\
\hline$\lambda=0.992$ & 39970 & 0.73 & 0.95 & 1.22 \\
\hline$\lambda=0.990$ & 37770 & 0.60 & 3.06 & 1.00 \\
\hline
\end{tabular}

The measurement results related to the settling time analysis are reported in Table III. From its analysis it is possible to highlight that:

a) a value of $\lambda$ equal to 0.999 is not suitable to the analysis because it cannot mitigate the memory effect of the sequential Burg method and as a consequence it continues estimating the initial tone at $10 \mathrm{MHz}$ even if it is not present, causing a frequency error ever greater than $1.5 \%$ during the second, the third and the fourth hop;

b) as experienced in the previous section $t_{s e t, A}, t_{s e t, B}$ and $t_{s e t, C}$ are influenced by the forgetting factor value, in particular the proposed method seems to reduce its convergence time when $\lambda$ value decreases from 0.999 to 0.99 .

As regard mean convergence frequency error $\left(\mu_{e f}\right)$ and the standard deviation of the convergence frequency error $\left(\sigma_{e f}\right)$, they have been evaluated in the time interval elapsed from the end of the settling time and the instant strictly before the tone makes a new hop.

From the analysis of the results, reported in Table IV, the following considerations can be drawn:

a) the mean frequency error obtained when the analyzing signal is a single tone at a fixed frequency is ever lower than $72 \mathrm{kHz}$, very similar to that experienced in the previous section;

b) the experimental standard deviation $\left(\sigma_{e f}\right)$ seems to be influenced in inverse proportion by $\lambda$, it is important to consider that the frequency resolution of the analysis is $24 \mathrm{kHz}$;

c) as experienced in the previous section, a value of $\lambda$ inside the range $0.992-0.998$ seems to grant a good trade-off between measurement accuracy and convergence time.

In Fig.4, as an example, the obtained spectrograms for three values $\lambda$ are reported. In particular the values considered are $\lambda=0.999, \lambda=0.996$ and $\lambda=0.900$. The information related to the amplitude of the PSD is coded by the color and the measurement unit is $\mathrm{dBm}$.

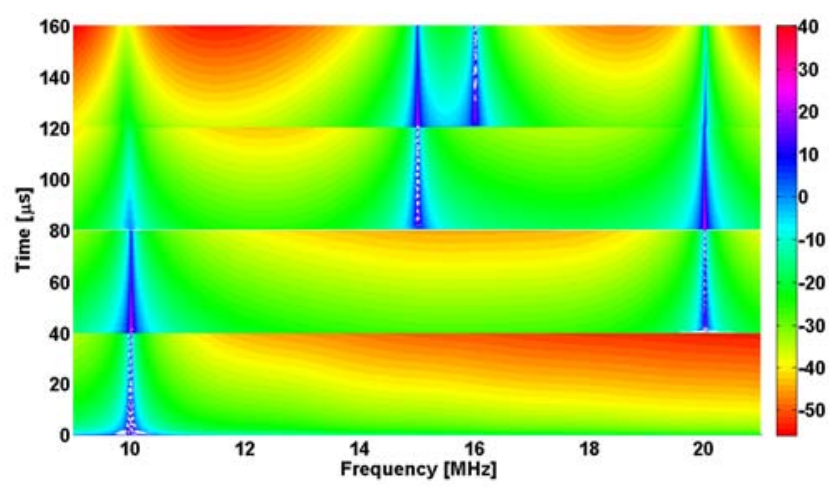

(a) $\lambda=0.999$

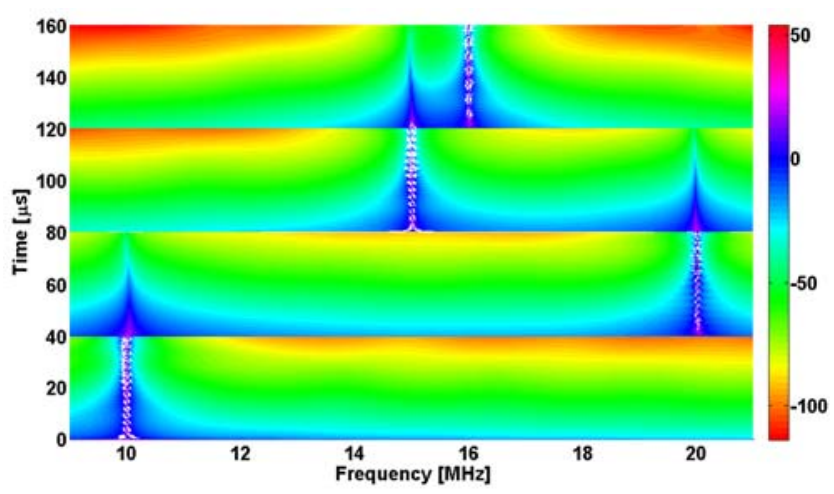

(b) $\lambda=0.996$

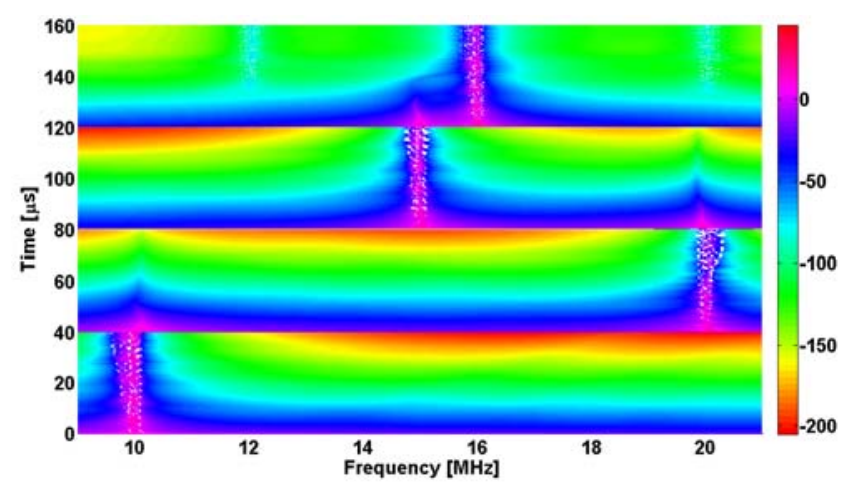

(c) $\lambda=0.990$

Fig.4. The obtained spectrograms, evaluated considering three values of $\lambda$.

From the analysis of these results the following consideration can be drawn:

a) all the measures are characterized by a good signal to noise ratio (SNR);

b) measurement results, obtained when $\lambda=0.999$ is involved, are affected by the memory effect that does not allow a good frequency tracking;

c) a value of $\lambda$ equal 0.99 even if it is less affected by memory, it seem to be not adequate for this analysis, showing an instability in frequency tracking performance;

d) an intermediate value of $\lambda$ equal to 0.996 seems to present negligible memory effects and a good frequency tracking performance. 
MEASUREMENT SCIENCE REVIEW, Volume 11, No. 1, 2011

Table IV. Measurement Performance at Steady-State. Mean Convergence Frequency Error and Standard Deviation of the Convergence Frequency Error Versus Forgetting Factor.

\begin{tabular}{|c|c|c|c|c|c|c|c|c|}
\hline \multirow{3}{*}{$\begin{array}{c}\text { Forgetting } \\
\text { factors } \lambda\end{array}$} & \multicolumn{2}{|c|}{$t_{\text {set, }, i n}<t<40 \mu s$} & \multicolumn{2}{|c|}{$t_{\text {set }, \mathbf{A}}<\boldsymbol{t}<\mathbf{8 0} \mu \mathrm{s}$} & \multicolumn{2}{|c|}{$t_{s e t, \mathrm{~B}}<t<120 \mu \mathrm{s}$} & \multicolumn{2}{|c|}{$t>t_{\text {set }, C}$} \\
\hline & $\mu_{e f}$ & $\sigma_{e f}$ & $\mu_{\text {ef }}$ & $\sigma_{e f}$ & $\mu_{e f}$ & $\sigma_{e f}$ & $\mu_{e f}$ & $\sigma_{e f}$ \\
\hline & \multicolumn{2}{|c|}{ Mef } & \multicolumn{2}{|c|}{ [kHz] } & \multicolumn{2}{|c|}{ [kHz] } & \multicolumn{2}{|c|}{ [kHz] } \\
\hline$\lambda=0.999$ & 25 & 16 & N/A & N/A & N/A & N/A & N/A & N/A \\
\hline$\lambda=0.998$ & 25 & 17 & 29 & 12 & 24 & 12 & 26 & 12 \\
\hline$\lambda=0.996$ & 22 & 30 & 23 & 22 & 17 & 25 & 17 & 23 \\
\hline$\lambda=0.994$ & 19 & 38 & 20 & 35 & 46 & 35 & 16 & 35 \\
\hline$\lambda=0.992$ & -72 & 78 & 32 & 51 & 14 & 48 & 13 & 47 \\
\hline$\lambda=0.990$ & -3.5 & 46 & 27 & 62 & -9.5 & 55 & 5.6 & 54 \\
\hline
\end{tabular}

\section{CONCLUSIONS}

An innovative digital processing method for spectrum management and monitoring has been designed. It is based on a modified version of the sequential Burg algorithm in order to mitigate its memory effects and giving the ability to track the frequency variation of the analyzing signal during the observation period.

In particular the Burg cost function has been modified by using an exponentially growing window with the aim of weighing the input samples giving more importance to the recent samples and attenuating the effects of the past ones. This solution avoids the need to store samples in the memory. This feature makes it a suitable candidate to be implemented on a cost-effective digital signal processing platform (DSP-based or FPGA-based). A preliminary characterization campaign has been designed to analyze the tracking capabilities and the measurement accuracies of the method. To this aim a test signal, characterized by a single tone that makes a little frequency hop and after that starts a linear sweep and then it carries out a deep frequency hop stopping at a fixed frequency, has been considered. This choice allows simulating some conditions that are common in practice, especially in spectrum monitoring activities. In particular, a good settling time, not influenced by $\lambda$, has been experienced when a single frequency tone is tracked. Excellent convergence time has been evaluated when the method tracks a signal that after a little hop start a linear frequency sweep or when the signal makes a deep frequency hop and then stop its frequency variations. Small mean frequency error, ever lower than $0.33 \%$ when the test signal is characterized by a single frequency tone, has been evaluated.

The performance assessment carried out on a different signal (characterized by several frequency hops) has confirmed that the proposed method shows a good trade-off between the measurement accuracy and the convergence time when $\lambda$ falls in the range $0.992 \div 0.998$. Future activities will be addressed to implement the proposed method on a cost effective hardware platform and to make a comparison with competitor measurement instruments available on the market.

\section{REFERENCES}

[1] Textronix Inc. (2007). Application Note "Advanced Spectrum Management with the RSA6100A Series Real-Time Spectrum Analyzer". Retrieved March 4, 2008, from Tektronix web site http://www.tek.com.

[2] Textronix Inc. (2007). Application Note "RF Signal Monitoring and Spectrum Management Using the Tektronix RSA3000B Series Real-Time Spectrum Analyzer". Retrieved March 4, 2008, from Tektronix web site http://www.tek.com.

[3] Luther, W.A. (2003). Spectrum management in the global village. In IEEE International Symposium on Electromagnetic Compatibility 2003, Vol. 2, 11-16 May 2003. Istanbul, Turkey, 701-704.

[4] Tektronix Inc. (2006). RSA3408A 8 GHz Real-Time Spectrum Analyzer User Manual. Beaverton, OR: Tektronix Inc.

[5] Erickson, D. (2007). Improving spectrum masurement and monitoring in commercial wireless applications. Agilent Meas. J., 2, 14-19. Retrieved May 30, 2007, from Agilent Technologies web site http://www.agilent.com.

[6] Agilent Technologies Inc. (2009). Datasheet Agilent W1314A Multi-band Wireless Measurement Receiver. Retrieved April 30, 2009, from Agilent Technologies web site http://www.agilent.com.

[7] TCI International Inc. (2007). Datasheet TCI Model 745 spectrum monitoring system. Retrieved July 17, 2008, from TCI International web site http://www.tcibr.com.

[8] Marple, L. (1987). Digital Spectral Analysis with Applications. Englewood Cliffs, NJ, USA: PrenticeHall.

[9] Angrisani, L., Capriglione, D., Ferrigno, L., Miele, G. (2008). Power measurements in DVB-T systems: New proposal for enhancing reliability and repeatability. IEEE Trans. Instrum. Meas., 57 (10), 2108-2117.

[10] Angrisani, L., Capriglione, D., Ferrigno, L., Miele, G. (2008). Sequential parametric spectral estimation for power measurements in DVB-T systems. In IEEE Instrumentation and Measurement Technology Conference, 12-15 May 2008. Victoria, BC, Canada, 314-319. 
[11] Angrisani, L., Capriglione, D., Ferrigno, L., Miele, G. (2009). Power measurement in DVB-T systems: On the suitability of parametric spectral estimation in DSP-based meters. IEEE Trans. Instrum. Meas., 58 (1), 76-86.

[12] Burg, J.P. (1967). Maximum entropy spectral analysis. In 37th Meeting Society of Exploration Geophysicists, 31 Oct. 1967. Oklahoma City, USA.
[13] Kay, S.M., Marple, S.L. (1981). Spectrum analysis-A modern perspective. In Proc. of the IEEE, 69 (11), 1380-1419.

[14] Srinath, M.D., Viswanathan, M.M. (1975). Sequential algorithm for identification of parameters of an autoregressive process. IEEE Trans. Automat. Contr., 20 (4), 542-546.

[15] Manolakis, D.G., Ingle, V.K., Kogon, S.M. (2005). Statistical and Adaptive Signal Processing. Norwood, MA, USA: Artech House. 